\title{
Design and performance analysis of transmission line-based nanosecond pulse multiplier
}

\author{
RISHI VERMA, A SHYAM and KUNAL G SHAH \\ Institute for Plasma Research, Gandhinagar 382 428, India \\ e-mail: rverma@ipr.res.in
}

MS received 19 August 2005; revised 9 June 2006

\begin{abstract}
Conventionally, Marx generators are used for the production of short duration, high voltage pulses but since many discharge gap switches are utilized for stepping up the voltage, there are many disadvantages. Here, an alternative and much simpler technique for the multiplication of nanosecond high voltage pulses has been presented in which multiplication takes place by switching single spark gap providing voltage gain of ' $n x V$ ' where $n$ is the subsequent number of stages. Stepped up high voltage pulse with fixed voltage gain of defined shape with fast rise time and good flat top is produced without using additional pulse-forming network. Its operation has been made repetitive by switching single spark gap. Multipurpose use, low cost, small size, light weight (weighing less than $50 \mathrm{~kg}$ ) and portability are the additional benefits of the system. The reported nanosecond pulser has been made by cascading three stages of Blumlein. To cross check its performance the parasitic impedance of the system has been evaluated to realize its adverse effect on the voltage gain and pulse shape. Also its operation has been simulated by PSPICE circuit simulator program and good agreement has been obtained between simulated and experimental results. Applications of this pulse generator include X-ray generation, breakdown tests, ion implantation, streamer discharge studies and ultra wideband generation, among others.
\end{abstract}

Keywords. Blumlein; spark gap switch; parasitic impedance; transmission line.

\section{Introduction}

In the pulser that we have developed three stages of coaxial Blumlein consisting of six pieces of transmission lines are charged in parallel and then discharged synchronously in series to the matched load by using a single switch. The output pulse is the sum of two discrete pulses from each transmission line finally resulting in a gain of six times the charging voltage (Rossie et al 2002).

The switching has been done by pressurized high voltage spark gap, as this is highly reliable and rugged even with repetitive switching. The pulser has been constructed using standard coaxial cables as they have well-defined impedance, transmission line characteristic, and reliability. Also cables are straightforward to work with, as use of coaxial cables also 


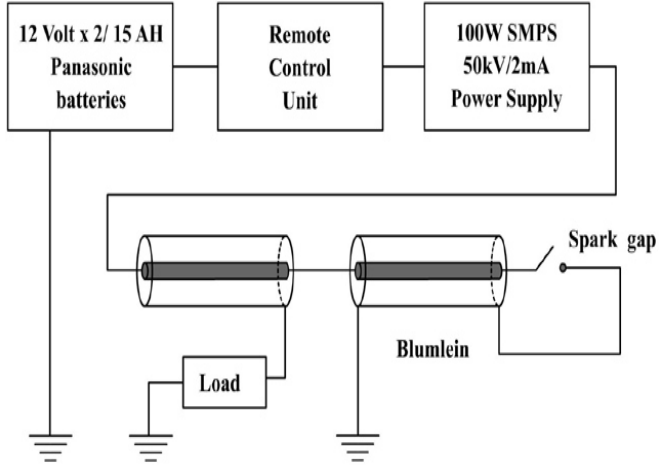

Figure 1. Basic components of Blumlein pulser.

provides significant flexibility in obtaining various pulse widths, voltages and impedance transformations. They are also relatively inexpensive and easy to replace in the event of breakdown. Charging of the coaxial transmission line is done using 100W SMPS powered by 24V DC Panasonic batteries, which makes the system portable. It converts the DC input to $50 \mathrm{kV} / 2 \mathrm{~mA}$ as output. The total weight of power supply including batteries is less than $3 \mathrm{~kg}$. A block diagram of the basic parts of the pulser is shown in figure 1 .

\section{Pulser design}

The Blumlein configuration presented here produces higher output voltages without overstressing the cable. In this cascaded arrangement, higher voltage gains are achieved by charging $n$ number of transmission lines in parallel and then connecting their outputs in series to produce the amplified output. The schematic of a typical single-stage Blumlein configuration is seen shown in figure 2 (Somerville et al 1990).

As seen, the single-stage Blumlein consists of two sections of transmission lines of equal length and the same characteristic impedance. Here, the line is first charged up to voltage $V$. When the switch $S$ is closed the wave starts and travels along the first section of the transmission line. After transition time $t_{o}$, a second wave is excited which travels along another

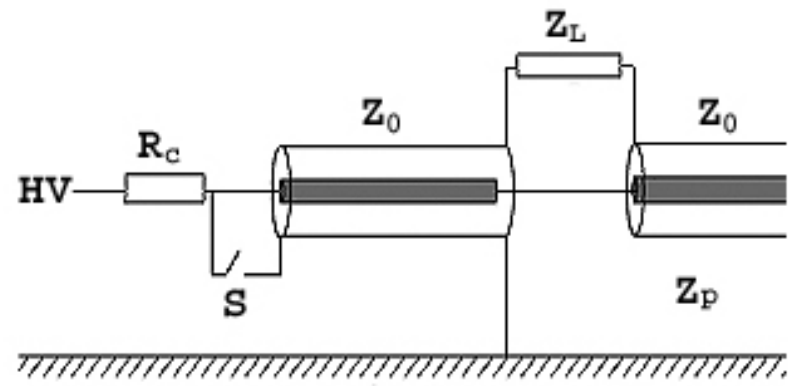

GROUND
Figure 2. Schematic for singlestage Blumlein generator. 
section of the line. Both waves are reflected at the inhomogeneities of the line. The potential difference across the matched load produced by these waves appears as a square pulse, which has an amplitude of $V$ and time duration $2 t_{o}$. The results of such theoretical analysis are mathematically represented as below (Somerville et al 1990).

The output voltage of Blumlein is

$$
V_{o}=2 V Z_{L} /\left(Z_{L}+2 Z_{O}\right) .
$$

Hence, for the matched load condition

$$
Z_{L}=2 Z_{o}, V_{o}=V,
$$

whereas in open load condition

$$
Z_{L} \gg Z_{o}, V_{o}=2 V .
$$

Transition time along one section of line is given by

$$
t_{o}=l \sqrt{\epsilon r} / c
$$

where,

$l=$ length of each section of transmission line,

$\in r=$ dielectric constant of the insulation used,

$c=$ velocity of light.

Thus, the pulse width $T$ is twice the time taken by the electromagnetic wave to travel the length of the coaxial line in the dielectric medium, i.e.

$$
T=2 t_{o}
$$

Characteristic impedance of the transmission line is given by

$$
Z_{0}=(L / C)^{1 / 2},
$$

where, $L$ and $C$ are the inductance and capacitance of the coaxial line per unit length.

The energy per pulse $\left(E_{p}\right)$, delivered to the load $\left(Z_{L}\right)$ is

$$
E_{p}=T x V_{o}^{2} / Z_{L} .
$$

Here, $T$ is the pulse length and $V_{o}$ is the amplitude.

\section{Cascaded Blumlein}

The schematic diagram of the three-stage cascaded Blumlein configuration is shown in figure 3. Each stage is constructed by connecting two transmission lines in series. The principle of operation of the device remains the same as mentioned, i.e. all the three stages are charged in parallel and discharged synchronously in series to the load by a single spark gap switch at the device input. The series addition which persists for twice the length of the line propagation time leads to an output voltage of $N V$, where, $N$ is the number of stages employed and V is the voltage up to which the line is charged (Smith 2002). 


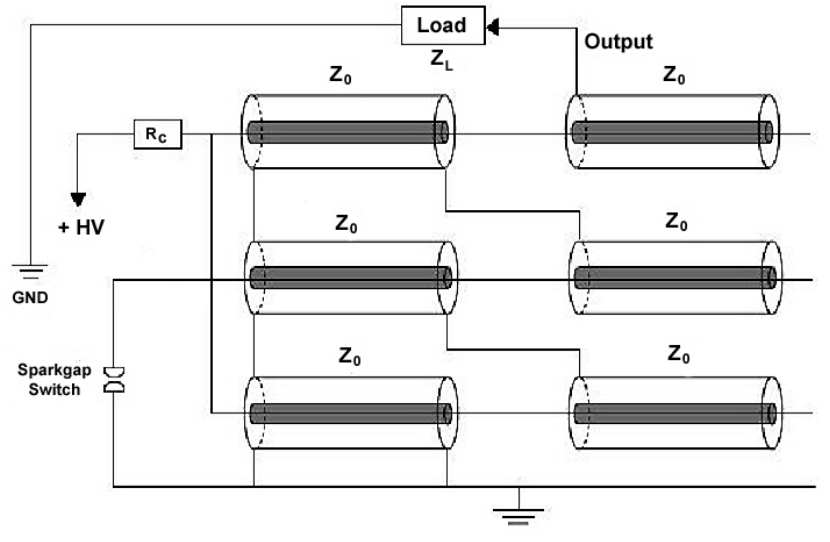

Figure 3. Schematic of threestage cascaded pulser.

For matched load conditions, load impedance should be equivalent to pulser output impedance, i.e.

$$
Z_{L}=n \times Z_{o}
$$

Here, $n$ is the total number of transmission lines used in the cascade.

The output voltage in cascaded configuration is (Rossie et al 2002; Soto \& Altamirano 1999)

$$
\begin{aligned}
& V_{o}=n x V / 2 \quad \text { (for matched load condition), } \\
& V_{o}=n x V \quad \text { (for open-ended condition) }
\end{aligned}
$$

For three-stage cascading (i.e. with six section of transmission lines having ideal gain of 3) nominal output voltage of $150 \mathrm{kV}$ across matched load of $300 \Omega$ requires maximum charging voltage of $50 \mathrm{kV}$. Under open-ended conditions, developed voltage is $300 \mathrm{kV}$. Here, each stage has been constructed using $5 \mathrm{~m}$ of coaxial transmission line. The cable chosen for this application is RG218, which has characteristic impedance of $50 \Omega$. The double transit time for $5 \mathrm{~m}$ length of cable implies pulse duration of $50 \mathrm{~ns}$. Each section of the transmission line contributes $50 \Omega$ to the total output impedance of the generator. Hence, with six sections in three stages the total output impedance of the pulser becomes $300 \Omega$. For matched load condition the maximum output is $150 \mathrm{kV} / 500 \mathrm{~A}$, delivering $75 \mathrm{MW}$ of peak power in $300 \Omega$ impedance, and for the open-ended case the maximum output is $300 \mathrm{kV}$.

As the distributed cable capacitance is of the order of $100 \mathrm{pF} / \mathrm{m}$, the total cascaded Blumlein capacitance is calculated as: $5 \mathrm{~m} \times 6 \times 100 \mathrm{pF} / \mathrm{m}=3 \mathrm{nF}$. At a charging voltage of $50 \mathrm{kV}$, the total energy stored in the system is $\sim 3.75$ joules. Now, though the power supply has maximum output voltage of $50 \mathrm{kV}$ and constant charging rate of $125 \mathrm{~J} / \mathrm{s}$, maximum pulse repetition rate of the order of $33 \mathrm{~Hz}$ can be obtained.

\section{Layout and construction}

The pulser has been constructed by stacking three Blumlein lines having cable lengths of $10 \mathrm{~m}$ each. Selected coaxial cables of RG218 grade have characteristic impedance of $50 \Omega$ 


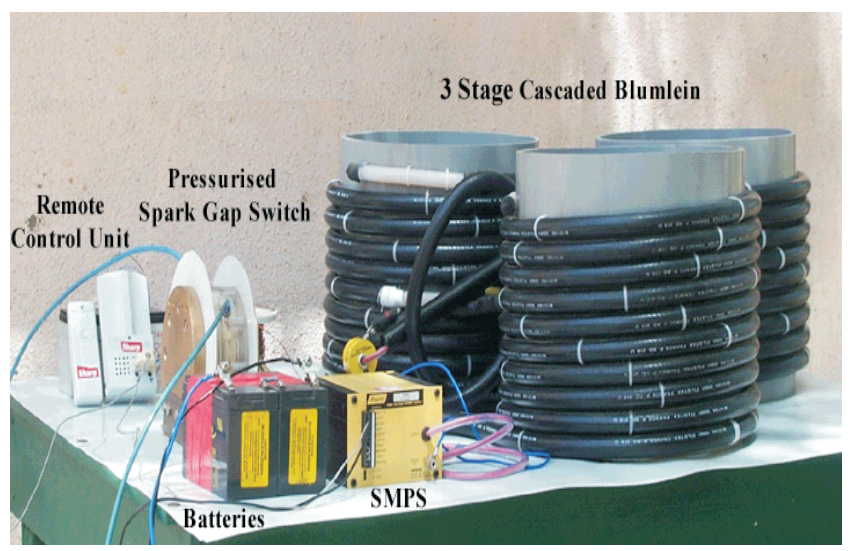

Figure 4. Experimental layout of the cascaded pulser.

and are rated for $100 \mathrm{kV}$ (Tuema et al 1998). Polyethylene is used as insulation between the conductors having dielectric constant $(\in r)$ of $2 \cdot 3$. From each line, the outer sheath of the cable has been removed from the centre to create two sections of transmission lines of $5 \mathrm{~m}$ lengths, with a common core conductor. Then, the outer braid at both ends of each transmission line module is removed for a distance sufficiently long to enable operation of the pulser at charging voltages up to $50 \mathrm{kV}$ without flash over. One end of all the three Blumlein is terminated to a single spark gap switch and the charging terminal is directly connected to the upper electrode of pressurized spark gap switch. The lower electrode is the ground reference of the system. Conventionally, the other end of each line has been left open-ended at the non-switching end. The winding arrangement along with experimental layout has been shown in figure 4 .

In order to increase the device gain efficiency (Somerville et al 1990; Rossie et al 2002) and make the pulser compact, individual stages are wounded on insulated PVC former of diameter $30 \mathrm{cms}$. Since the outer diameter of RG218 coaxial cable is $2.2 \mathrm{cms}$, winding length of about $25 \mathrm{cms}$ is obtained for the cable length of $10 \mathrm{~m}$ each. It is to be noted that for reducing the risk of 'dielectric cold flow', former diameter of at least 10 times the diameter of coaxial cable (Gupta \& John 2002) has been used.

A low inductance two-electrode pressurized spark gap has been used as switch. The connection of coaxial cables with spark gap electrodes is done through parallel plate transmission lines, in order to reduce the residual system inductance as small as possible. The switch is capable of working up to pressures of 4 bars absolute and can withstand maximum hold-off voltage up to $50 \mathrm{kV}$. For producing perfect square pulse, non-inductive resistance $\left(Z_{L}\right)$ of $300 \Omega$ has been used as load impedance. It is equivalent to the generator output impedance and is connected between the final output of the cascade and ground. The ON/OFF switching of the power supply input i.e. $24 \mathrm{~V} \mathrm{DC}$ is done through RF transmitter/receiver sets so that the pulser can be remotely operated within a range of $25 \mathrm{~m}$.

\section{Output pulse waveform}

An oscillogram of the output voltage and current pulse of the cascaded Blumlein pulser, when charged up to voltage of $23 \mathrm{kV}$ is shown in figure 5 .

The voltage was measured to have a value of $60 \mathrm{kV}$ across the matched load of $300 \Omega$ using 1000X RC compensated high voltage probe (make - North Star, model - PVM1) having 


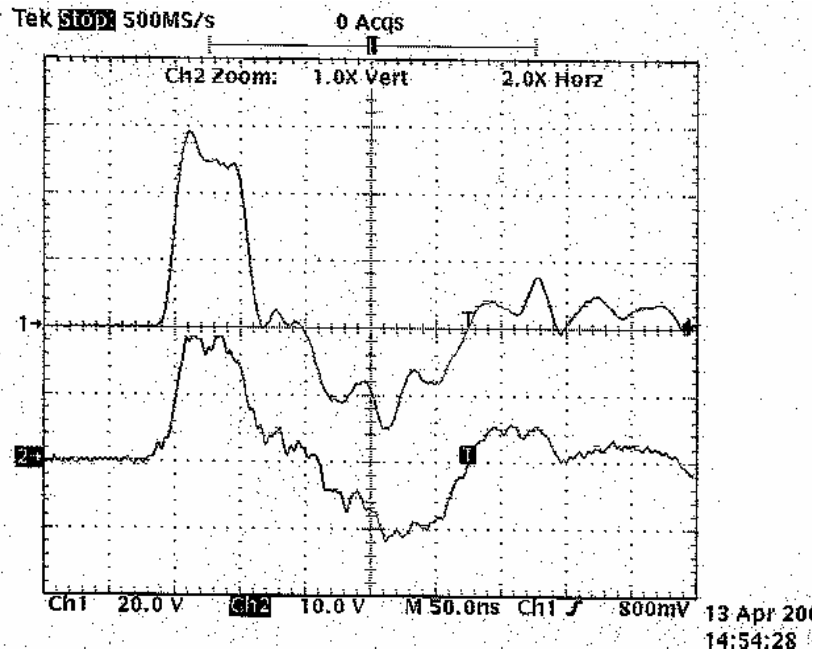

Figure 5. Oscillogram of output voltage (channel $-1,20 \mathrm{kV} / \mathrm{div}$ ) and current pulse (channel -2, $100 \mathrm{~A} /$ div) when pulser is charged up to $23 \mathrm{kV}$.

bandwidth of $90 \mathrm{MHz}$. This results in producing a system gain of 2.6 which corresponds to voltage gain efficiency of $87 \%$. The current pulse is measured to have value of $200 \mathrm{~A}$, using current transformer (make - Ion Physics, model - MG10) having bandwidth of $35 \mathrm{MHz}$. The flat-top duration of the pulse is $50 \mathrm{~ns}$ with rise time of better than $10 \mathrm{~ns}(10-90 \%)$. For the generator capacitance of $3 \mathrm{nF}$ at $23 \mathrm{kV}$ charging, the energy per shot is $0.8 \mathrm{~J}$ with peak power of $12 \mathrm{MW}$ developed during the pulse. Repetitive operation of the pulser in the burst mode (MacGregor et al 1998) has been shown in figure 6.

\section{Performance analysis}

Regarding the pulser configuration (as shown in figure 2), voltage gain efficiency of the system is reduced by presence of parasitic line impedance $\left(Z_{p}\right)$ (Somerville et al 1990). It exists between the outer part of the shield and ground.

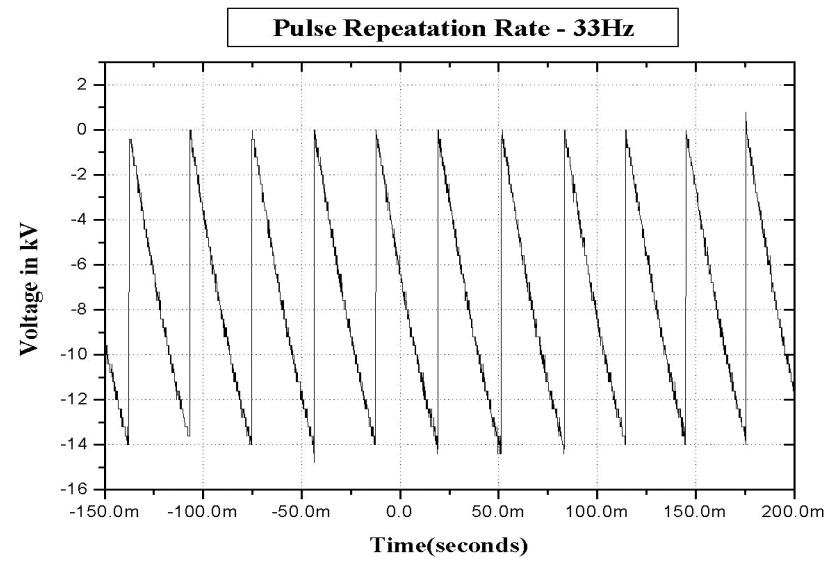

Figure 6. Repetitive operation of pulser with $\mathrm{N}_{2}$ /air at pressure of 1 bar in burst mode at PRR of $33 \mathrm{~Hz}$. $\left(1 \mathrm{bar}=10^{5} \mathrm{~N} / \mathrm{m}^{2}\right.$ or $\left.10^{5} \mathrm{~Pa}\right)$. 


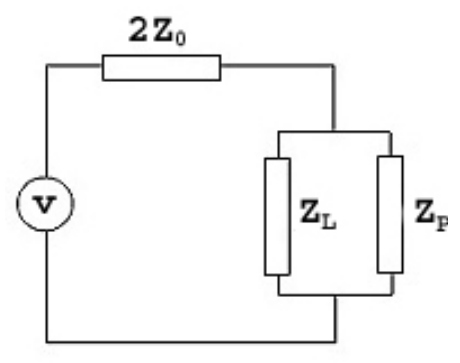

Figure 7. Schematic for equivalent circuit considering parasitic line impedance.

Though, the parasitic impedance $\left(Z_{p}\right)$ appears parallel with load impedance $\left(Z_{L}\right)$. Hence, it reduces the gain of the system. In addition to affecting the voltage gain efficiency, it also causes trailing reflections after the square pulse which can be observed in the oscillogram. Reflections are also observed due to dissimilar transmission line transit times. Thus to achieve higher system efficiency, the parasitic impedance $\left(Z_{p}\right)$ should be much larger than characteristic impedance $\left(Z_{o}\right)$ of the transmission line. Moving the system well above ground plane and inductively winding the cable on an insulated former does this, so that impedance of outer part of the jacket remains high. These measures reduce drained current and consequently overall power loss of the device (Somerville et al 1990; Rossie et al 2002, 2003). in the output pulse waveform. The distorted tail portion of the pulse can be observed, it is because of impedance mismatch at the pulser output due to the presence of shielding cable impedances. In the case of cascaded Blumlein pulser, the system efficiency also reduces if the stacking arrangement leads to the formation of secondary transmission line between cable braids. To avoid such effects, we have inductively wound coaxial cables on the PVC former and integrated the system, keeping each stage separate.

The pulse-rise time consideration mainly depends upon the residual system inductance and spark gap design (Tuema et al 1998). The system inductance should be kept as small as possible. Rise time as a function of spark gap design depends on distance between the spark gap electrodes and applied electric field across the gap. Hence to reduce the rise time, it is necessary to reduce the spacing between electrodes. This effectively increases the electric field and decreases the resistive phase of the closure time. In our case, spark gap spacing between the electrodes has been kept at $10 \mathrm{~mm}$ and the achieved rise time is better than $10 \mathrm{~ns}$.

The repetitive operation of the pulser is mainly dictated by power supply charging rate capability and type of gas used in spark gap switch for fast recovery (Mcgreagor et al 1998). Use of high current, fast charging power supply and stronger electronegative gases in spark gap could result in pronounced increase of pulse repetition frequency.

\section{Evaluation of parasitic impedance}

Evaluation of parasitic impedance is necessary in order to assess the voltage gain of the pulser (Rossie et al 2002, 2003). It is equivalent to the shield cable impedance given by $(L / C)^{1 / 2}$, where $L$ and $C$ are inductance and capacitance of the cables wound inductively on an insulated former. On the basis of constructional parameters equivalent inductance and capacitance with respect to ground plane are given as (Rossie et al 2003)

$$
\begin{aligned}
& L=4 \pi^{2} \mu_{r} n^{2} r_{L}^{2} 10^{-7} / \ell \text { (Henry), } \\
& C=24 \times 10^{-12} \ell /\left[\log \left\{2 h / r_{c}\right\}\right] \text { (Farads). }
\end{aligned}
$$


Combining (9) and (10) gives the expression for parasitic impedance evaluation (Rossie et al 2002, 2003a),

$$
Z p=(L / C)^{1 / 2} .
$$

Here $\mu_{r}$ is the relative magnetic permittivity, $h$ is the spacing between the outer part of the coaxial winding, $\ell$ is the winding length, $r_{L}$ and $r_{c}$ are respectively the mean and outer radii of the winding and $N$ is the number of turns. Using $5 \mathrm{~m}$ length of the cable wound on a PVC former with diameter $30 \mathrm{~cm}$ (i.e. $a=0.15 \mathrm{~m}$ ) gives five turns. Since the outer diameter of the cable is $22 \mathrm{~mm}$, winding length $(\ell)$ of about $25 \mathrm{~cm}$ is obtained for the cable length of $10 \mathrm{~m}$. Though the cable outside diameter is much smaller than the PVC former diameter, the approximation $a \approx r_{L} \approx r_{c}$ can be made (Rossi et al 2003c).

As the spacing between the outer part of the coaxial winding and ground plane is $0.5 \mathrm{~m}$, calculated inductance and capacitance from (9) and (10) are $20.46 \mu \mathrm{H}$ and $7.28 \mathrm{pF}$ respectively. Thus, estimated parasitic impedance of the system is $1.673 \mathrm{k} \Omega$. For increasing parasitic impedance, spacing between the two adjacent turns on the PVC former should be increased in order to keep the stray capacitance of the system as small as possible.

\section{PSPICE simulation}

In order to investigate the reflections associated with the waveform as shown in figure 5, analysis and realization of the pulser has been done using the circuit simulator program PSPICE (Rossie et al 2003b). Modelling of the pulser has been done in two stages. In the first stage, pulse generator is modelled by ideal coaxial lines with characteristic impedances of $50 \Omega$, one way transit time of $25 \mathrm{~ns}$ and connected to matched load impedance of $300 \Omega$. In the modelled circuit as shown in figure 8 , the effect of parasitic impedance has been neglected.

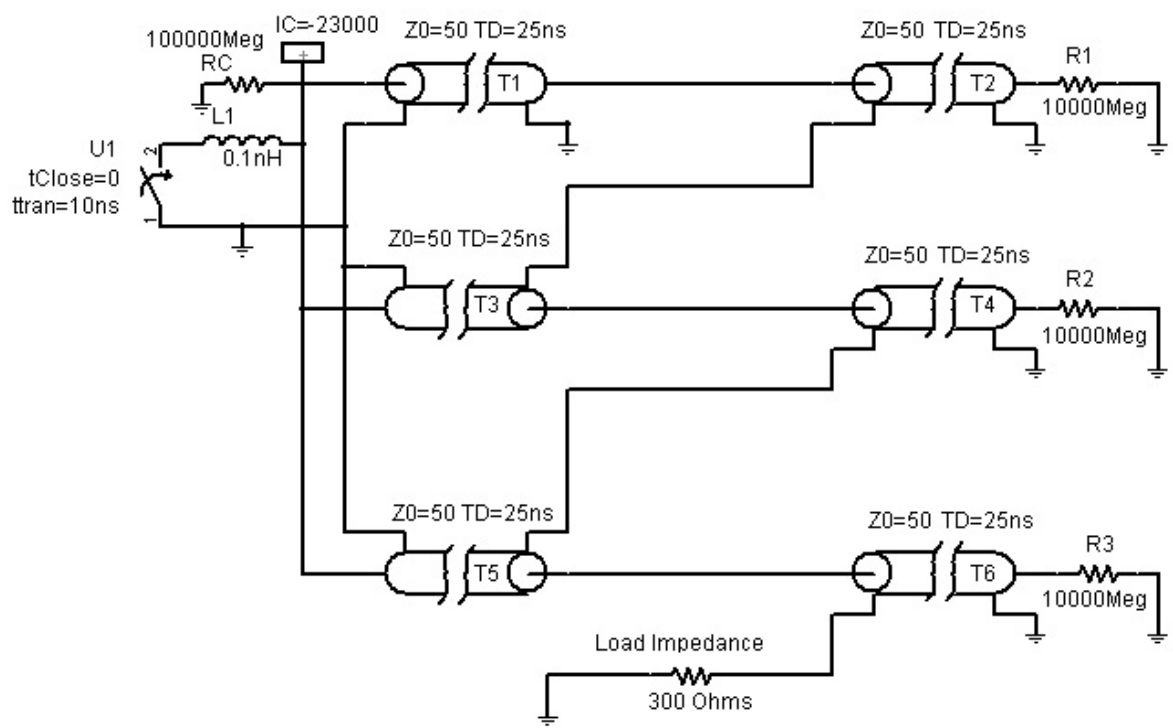

Figure 8. PSPICE model of ideal Blumlein pulser. 


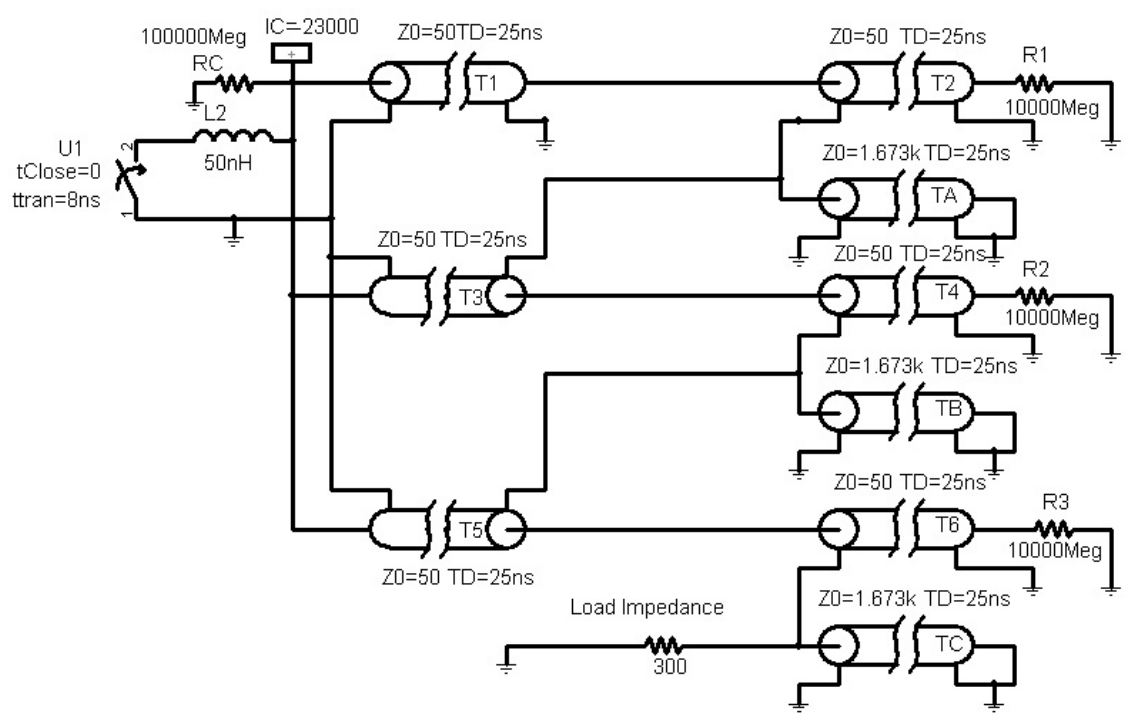

Figure 9. Modelling of practical $n$-stage cascaded Blumlein considering losses.

To avoid node-listing problems in PSPICE simulation open-ended transmission lines have been terminated through high value resistors in the range of giga ohms (Rossie et al 2003b). Transition time (i.e. 'ttrans') of the closing switch in the PSPICE schematics decides the pulse rise and fall times, which is of the order of $10 \mathrm{~ns}$.

In the second phase, modelling of the practical pulser has been done considering losses in coaxial lines. PSPICE model of $n$-stage cascaded Blumlein as shown in figure 9 has been developed taking into account all the factors affecting quality of output pulse, like impedance mismatch, switch inductance, parasitic impedance of cables, characteristic impedance of pulser and effect of unidentical sections.

Analysis of variation in line parameters has been studied through ideal and practical models presented in figures 8 and 9 respectively as given below.

\subsection{Analysis of impedance mismatch conditions}

In order to achieve maximum power transfer to load and avoid reflections, it is necessary that output impedance of the generator and load impedance should be equal. In order to predict the output pulse under load mismatch conditions, the ideal model (shown in figure 8) has been simulated for all the three possible conditions as follows.

(a) Load impedance $=$ generator impedance,

(b) Load impedance $>$ generator impedance,

(c) Load impedance $<$ generator impedance.

For simulating the 'mismatch' conditions generator to load impedance Ratio has been altered by $25 \%$ (i.e. for the case $Z_{L}>n Z_{o} ; Z_{L}=375 \Omega$ and for the case $Z_{L}<n Z_{o} ; Z_{L}=$ $225 \Omega$ ). The impact of 'mismatch ratio' has been simulated by altering the ratio to extent of $80 \%$ (i.e. for the case $Z_{L}>n Z_{o} ; Z_{L}=540 \Omega$ and for the case $Z_{L}<n Z_{o} ; Z_{L}=60 \Omega$ ). 


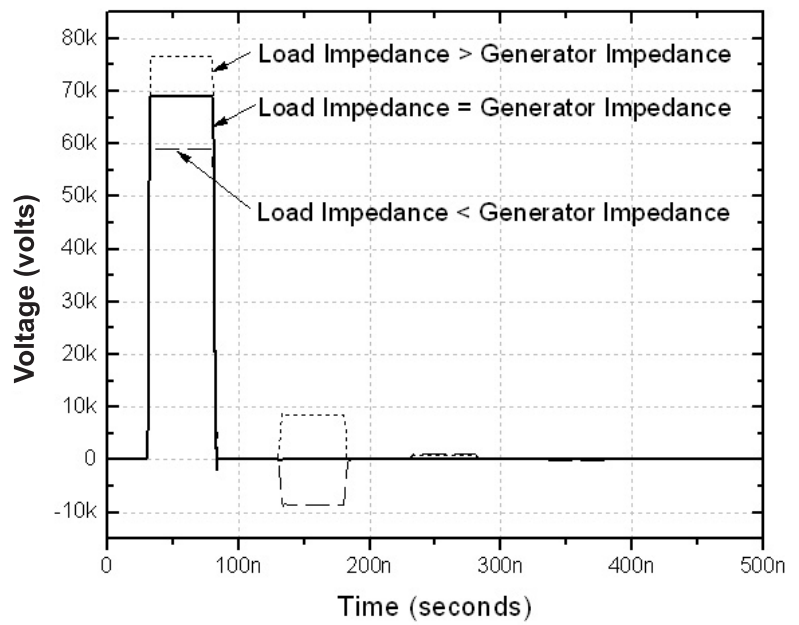

Figure 10. Impedance mismatch analysis output for a mismatch ratio of $25 \%$.

Upon observing the simulated outputs shown in figure 10 and 11, the effect of 'impedance mismatch' on pulser output has been summarized as below.

(a) When load impedance is perfectly matched with generator impedance, there are no reflections in the output and the voltage gain is as per theoretical prediction. As shown in graphs corresponding simulated output is a square pulse with amplitude of $69 \mathrm{kV}$ as expected for the duration of 50ns having, no reflections, with an initial charging voltage of $23 \mathrm{kV}$.

(b) Under impedance mismatch conditions, delivered pulse is followed by several reflections of same duration $2 t_{p}$ with gradually decreasing amplitude. As can be observed from the output (shown in figures 10 and 11), 'polarity' of oscillations/ reflections depends on ratio of load impedance to generator impedance. (a) If $Z_{L} / n Z_{o}>1$, oscillations are positive. (b) If $Z_{L} / n Z_{o}<1$, oscillations are negative.

(c) The 'decay rate' of oscillation/ reflections depends upon degree of mismatch. Upon comparing output pulses shown in figures 10 and 11, it can be seen that decay rate is

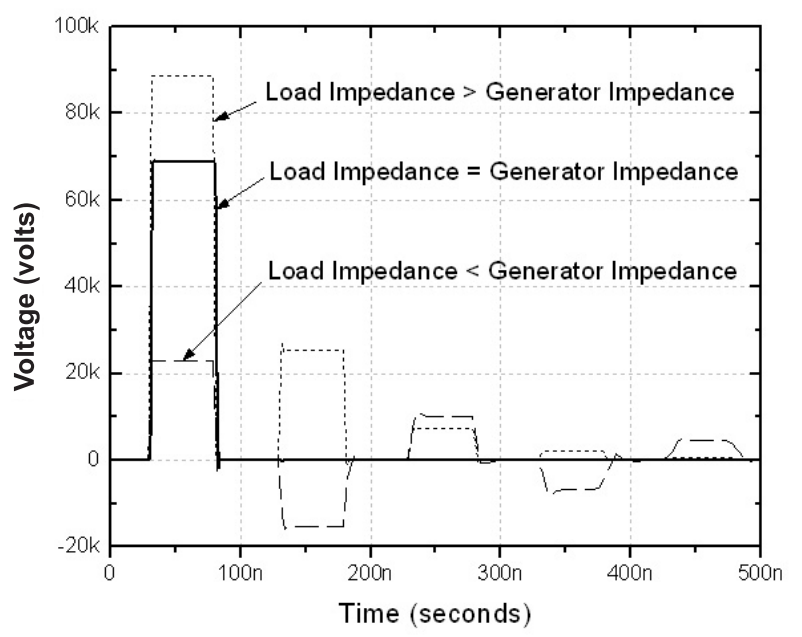

Figure 11. Impedance mismatch analysis output for a mismatch ratio of $80 \%$. 


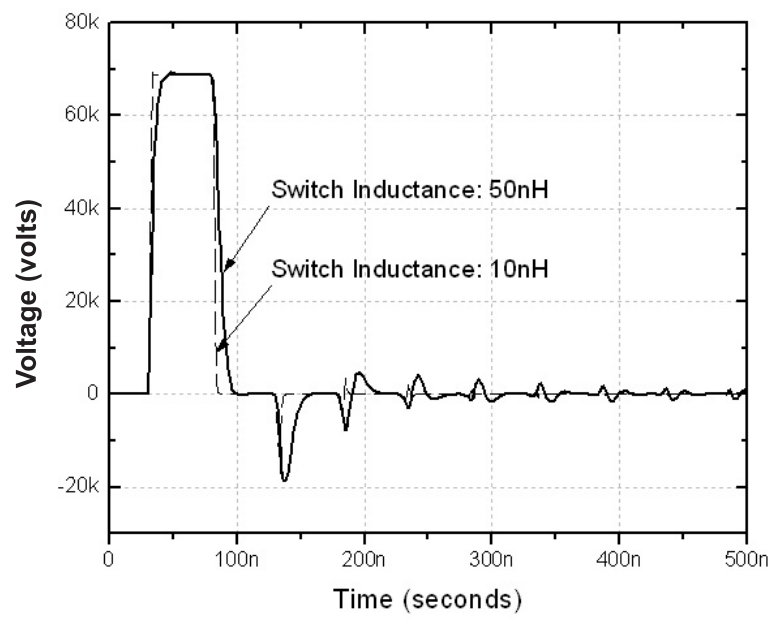

Figure 12. Comparative analysis of switch inductance effect (generator impedance: $300 \Omega$ ).

fast when mismatch ratio is $25 \%$ rather than when mismatch ratio is $80 \%$ of generator impedance.

(d) Impedance mismatch analysis also shows that voltage division between generator and load impedance determines the amplitude of output voltage.

\subsection{Analysis of switch inductance in reference with generator impedance}

Effect of switch inductance on pulser performance has been simulated using the ideal model shown in figure 8 . Ideal model has been simulated for the switch inductance of $10 \mathrm{nH}$ and $50 \mathrm{nH}$ for two different values of generator impedances i.e. $300 \Omega$ and $60 \Omega$ under matched load conditions. Corresponding simulated outputs have been shown in figures 12 and 13. It is observed that increase in switch inductance reduces the rise time of pulses and distorts the pulse shape introducing reflections as well. Upon comparing outputs in reference with

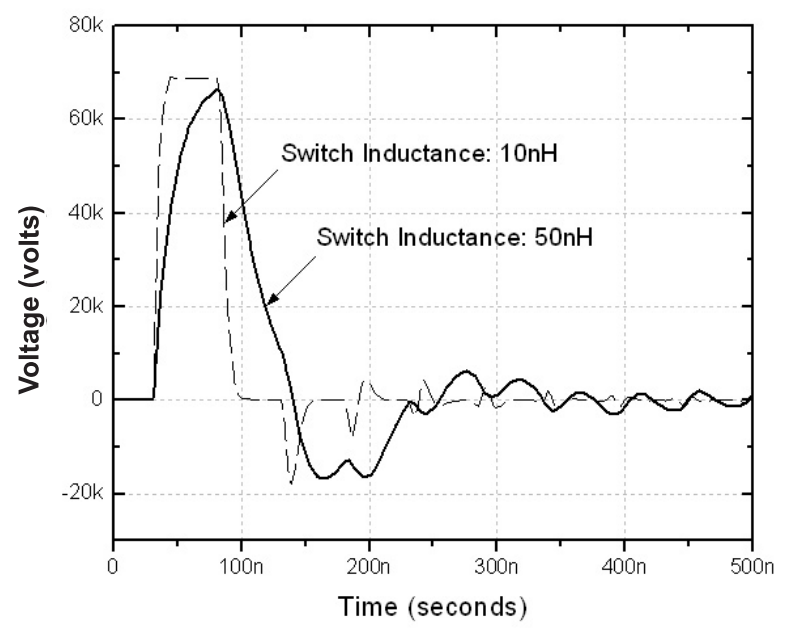

Figure 13. Comparative analysis of switch inductance effect (generator impedance: $60 \Omega$ ). 
generator impedance (refer figures 12 and 13), it is noticed that due to switch inductance corresponding reflection is much less when the generator impedance is $300 \Omega$ rather than when it is $60 \Omega$. This indicates that the effect of switch inductance is prominent when the generator impedance is less.

\subsection{Analysis of parasitic impedance effect in reference with generator impedance and pulse length}

Effect of parasitic impedance on pulser performance has been simulated using practical model shown in figure 9. As shown in the model parasitic impedance of the cable is formed between shielding and ground plane, causing degradation in external insulation. Parasitic lines are short-circuited at their input in the circuit by grounding inner conductors. By using the estimated values of $L$ and $C$, the model has been simulated with $Z_{p}=1.673 \mathrm{k} \Omega$ having one-way transit time of $25 \mathrm{~ns}$. Considering same parasitic effects simulation has been done for generators having output impedance of $300 \Omega$ and $60 \Omega$. As shown in figure 14, under matched load condition for generator impedance of $300 \Omega$ simulated output voltage and current pulse have amplitudes of $60 \mathrm{kV}$ and 200A respectively for an initial charging voltage of $23 \mathrm{kV}$. Voltage gain reduces from 3 to 2.6 due to presence of parasitic impedance. On comparing with the oscillogram shown in figure 5, we can conclude that the PSPICE modelling results are well in agreement with experimental results.

In order to analyse the effect of parasitic impedance in reference with generator impedance when the practical model has been simulated for generator impedance of $60 \Omega$ under matched load conditions, it is observed from the output (shown in figure 15) that reflections are prominent and the distortion in pulse shape is more. This fact indicates that low impedance generators are more susceptible to parasitic effects.

For analysing the effect of parasitic impedance with reference with pulse length, practical model has been simulated with same parasitic impedance having one-way transit time of $25 \mathrm{~ns}$ and $100 \mathrm{~ns}$. It is observed from output pulse (shown in figure 16) that parasitic impedance

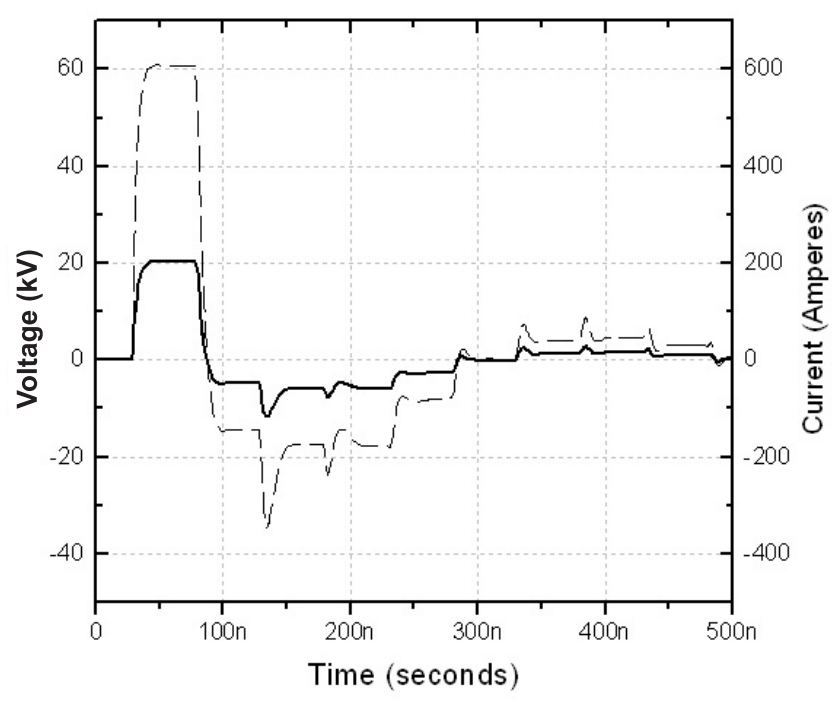

Figure 14. Simulated voltage and current output in practical Blumlein. 


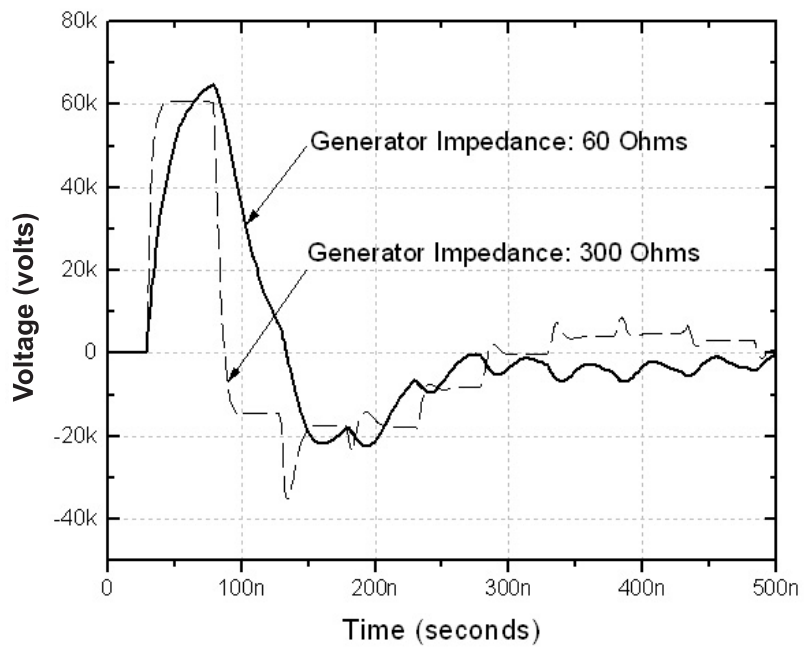

Figure 15. Comparative analysis of parasitic impedance effect versus generator impedance.

effect is prominent for short pulse lengths. Either way, in pulse profiles with longer rise times and pulse durations, the relative level of reflections is much less.

\subsection{Analysis of effect on pulse length due to un-identical sections of transmission line}

When there are non-identical sections in transmission line forming Blumlein, it basically affects the 'transit time' of pulse, which creates distortion in pulse shape. For analysing this fact, the ideal model (shown in figure 8 ) has been simulated with $20 \%$ of variation in either section of each stage of Blumlein i.e. one section of the Blumlein has transit time of $25 \mathrm{~ns}$ whereas another section has a transit time of $20 \mathrm{~ns}$. Corresponding outputs, when sections are identical and when they are non-identical has been shown in figure 17.

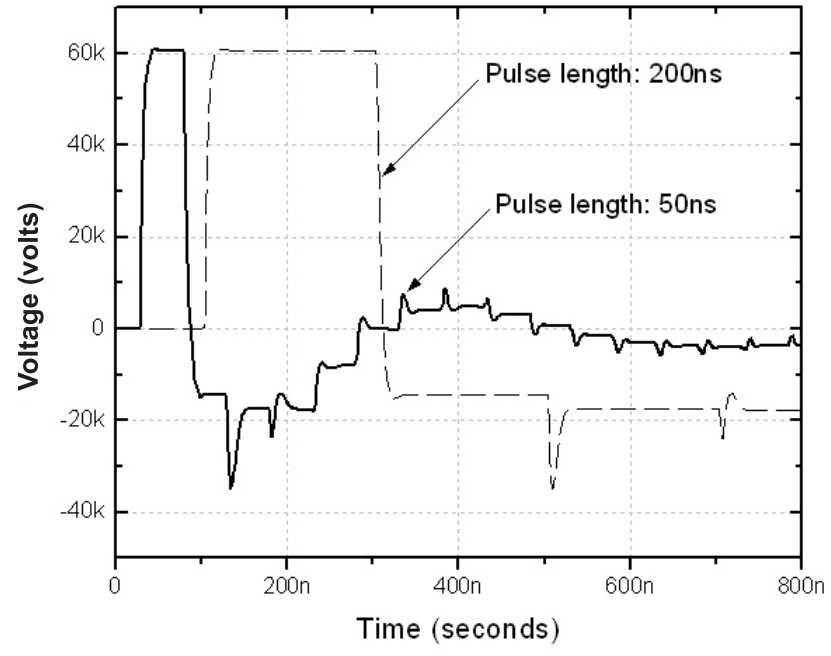

Figure 16. Comparative analysis of parasitic impedance effect versus pulse length. 


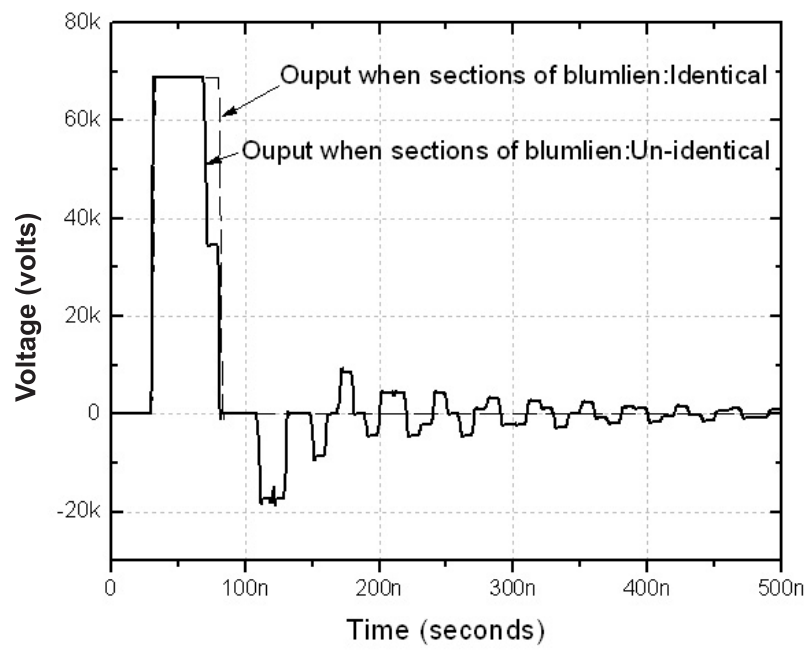

Figure 17. Simulated output voltage under ideal conditions.

The effect of non-identical sections can be observed on the output pulse duration as it contributes to distortion in pulse duration. Reflections are observed because of unbalancing in the system due to unequal transit times.

\section{Conclusion}

A cascaded Blumlein pulser has been developed using coaxial transmission lines. It has stacked considerably well having voltage gain efficiency of $87 \%$ giving short rise and fall times, flat-top with minimum ringing and consistent pulse shape even at high repetition rates. In the present configuration, the pulser can give maximum output voltage of $150 \mathrm{kV} / 500 \mathrm{~A}$ to a matched load impedance for duration of 50ns having rise and fall times better than $10 \mathrm{~ns}$.

Simulation of the generator shows that PSPICE modelling is well in agreement with experimental results. Thorough analysis of variation in transmission line parameters has been made. From the outputs obtained under various conditions, it is noticed that nanosecond kilovolt pulsers should be designed for high output impedance, so that the quality of output is least affected by degrading factors such as switch inductance and parasitic impedance. However, the fact remains that increase in output impedance of the generator limits the driving capacity of the pulser, hence optimization is required while designing. It has also been noticed that pulses of lower frequency, i.e. in pulse profiles with larger rise time and pulse duration, the relative level of reflection gets reduced significantly. This fact indicates pulse compression should only be done to the extent required, else tight control over degrading factors is required for avoiding distortions in output pulse.

The authors are grateful to all the members of the Pulsed Power Group for useful suggestions and kind cooperation in the fabrication and setting up of the multi-utility system. 


\section{References}

Gupta D K, John P I 2001 Design and construction of double-Blumlein HV pulse power supply. Sadhana 26: 475-484

MacGregor S J, Koutsoubis J M, Tumbull S M 1998 The design and operation of a compact high voltage, high pulse repetition frequency trigger generator. Meas. Sci. Technol. 9: 1899-1905

Rossi J O, Ueda M, Barroso J J 2002 Design of a 150 kV/300 A/100 Hz Blumlein coaxial pulser for long pulse operation. IEEE Trans. Plasma Sci. 30: 1622-1626

Rossi J O, Ueda M, Barroso J J 2003a A 150 kV/300 A/1 us coaxial Blumlein pulser. Proc. 14th IEEE Pulsed Power Conference, Dallas, pp. 661-664

Rossi J O, Ueda M, Barroso J J 2003b Design and pspicc simulation of a $150 \mathrm{kV} / 300 \mathrm{~A} / 1$ us coaxial pulse generator. Proc. 7th Brazilian Power Electronics Conference 1: 521-523

Rossi J O, Ueda M, Barroso J J 2003c Design and construction of a 150kV/300 A/1 us Blumlein pulser. Proc. WSEAS Trans. Syst. 1: 24-28

Smith P W 2002 Transient electronics and pulse circuit technology (New York: John Wiley \& Sons)

Somerville C, MacGregor S J, Farish O 1990 An efficient stacked-Blumlein HV pulse generator. Meas. Sci. Technol. 1: 865-868

Soto L, Altamirano L 1999 A pulse voltage multiplier. Rev. Sci. Instrum. 70: 1891-1892

Tuema F A, MacGregor S J, Fouracrc R A 1998 The design and performance of a low dependence, self-matched transmission line pulse generator. Meas. Sci. Technol. 9: 1989-1993 